While the total number of $\mathrm{AE}$ was comparable between the TOC cohort (58/100PY) and in the control cohort (54/100PY; RR 1.1; 95\% Cl 0.9-1.4), more serious AE (SAE) were reported with TOC (4.8/100PY compared to 1.4/100PY; RR 3.5; $95 \% \mathrm{Cl} 1$-10.9). Medically important infections and uveitis events were documented at significantly lower frequency in the TOC(0.8/100PY) than in the control cohort (4.1/100PY; RR 0.2; 95\% Cl 0.04-0.9). SAE with TOC were suicidal intent $(n=3)$, depression $(n=2)$, exacerbation of JIA, abscess, gastrointestinal infection, abdominal pain, colitis, bone surgery and fracture $(n=1)$. SAE in the control cohort were depression, osteomyelitis, gastrointestinal infection and superinfected eczema $(n=1)$. No significant differences regarding cytopenia and elevated transaminases were observed. No gastrointestinal perforation, no vascular event, no malignancy and no death occurred.

Conclusion: The efficacy of tocilizumab is comparable to that of alternative biologics. Tolerability was acceptable. As Tocilizumab was given as a second-line biologic in the vast majority of patients, comparisons between the cohorts have to be interpreted carefully. Observation in the registry is ongoing.

Disclosure of Interests: Ariane Klein Consultant of: Celgene, Toni Hospach: None declared, Frank Weller-Heinemann: None declared, Sandra Hansmann Consultant of: Advisory board Novartis Pharma, Jasmin Kuemmerle-Deschner Grant/research support from: Novartis, Sobi, Consultant of: Novartis, Sobi, Speakers bureau: Novartis, Sobi, Maria Fasshauer Consultant of: Shire, CSL Behring, Kirsten Minden Consultant of: GlaxoSmithKline, Sanofi, Speakers bureau: Roche, Ivan Foeldvari Consultant of: Novartis, Christoph Rietschel Consultant of: Pfizer, Abbvie, Novartis, Chugai, and Sobi, Tobias Schwarz: None declared, Ralf Trauzeddel: None declared, Markus Hufnagel: None declared, Dirk Foell Grant/research support from: Novartis, Sobi, Pfizer, Speakers bureau: Novartis, Sobi, Rainer Berendes: None declared, Gundula Boeschow: None declared, Prasad Oommen Consultant of: Novartis, Frank Dressler: None declared, Astrid Helling-Bakki: None declared, Gerd Horneff Grant/research support from: AbbVie, Chugai, Merck Sharp \& Dohme, Novartis, Pfizer, Roche, Speakers bureau: AbbVie, Bayer, Chugai, Merck Sharp \& Dohme, Novartis, Pfizer, Roche

DOI: 10.1136/annrheumdis-2020-eular.4363

\section{SAT0491 PSORIASIS ASSOCIATED WITH MONOCLONAL- ANTIBODY-TNF-A INHIBITORS VS. FUSION PROTEIN ETANERCEPT IN JUVENILE IDIOPATHIC ARTHRITIS PATIENTS - ANALYSIS OF THE BIKER REGISTRY}

A. Zimmer ${ }^{1}$, A. Klein ${ }^{1}$, G. Horneff ${ }^{1}{ }^{1}$ Asklepios Kinderklinik Sankt Augustin, Sankt Augustin, Germany

Background: Although efficacy of Tumor necrosis factor inhibitors (TNFi) for treatment of psoriasis is well established, patients may develop psoriasis for the first time while on TNFi as a paradoxical effect. Few data are available in patients with juvenile idiopathic arthritis (JIA).

Objectives: To analyze the incidence of psoriasis in TNFi - treated JIA patients and to identify associated factors.

Methods: Safety data from patients registered in the German Biologics registry (BIKeR) were analyzed. Cohorts of patients were grouped by treatment: any or multiple TNFi, single TNFi, biologics other than TNFi and no biologics (control group on methotrexate (MTX) only). TNFi-associated psoriasis was defined as incident diagnosis of psoriasis after starting a TNFi. Patients with personal history of psoriasis prior to TNFi therapy were excluded. Rates and events per
100 patient-years (PY) of exposure were calculated using AEs reported after first dose under therapy and under the age of 18 years. Rates were compared by X2-test, event rates by Wald test.

Results: A total of 4149 treatment episodes with TNFi (Etanercept, Adalimumab, Golimumab, Infliximab), with a total exposure time of 8437 PY, were identified. There were 676 treatments with a non-TNFi- biologic (Tocilizumab, Abatacept, Anakinra, Canakinumab) with a total exposure time of $1112 \mathrm{PY}$ MTX monotherapy was conducted in 1692 patients with a total exposure time of $3971 \mathrm{PY}$. In total, 31 patients were diagnosed with incident psoriasis on JIA-treatment (Table 1). The mean duration of therapy until incident psoriasis was $2.2( \pm 1.8)$ years. Multiple psoriatic skin manifestations were observed.

Psoriasis events were significantly more frequent in any or multiple TNFi compared to MTX-monotherapy, and specifically in the subgroup of TNF-antibody treatment (all) or Adalimumab compared to MTX or Etanercept (Table 2). Interestingly, psoriasis events were also observed with non-TNFi at high frequency. At occurrence of the event, patients exposed to biologics received MTX or steroids less frequently compared to the total patient cohort and had a higher JADAS10.

Table 2.

\begin{tabular}{lclccc}
\hline Reference & $\begin{array}{c}\text { Psoriasis event } \\
\mathrm{n} / 100 \mathrm{PY}\end{array}$ & Comparator & $\begin{array}{c}\text { Psoriasis event } \\
\mathrm{n} / 100 \mathrm{PY}\end{array}$ & Risk ratio & P-value \\
\hline Non-TNFi & 0.63 & TNFi & 0.27 & $0.4(0.2-1.0)$ & 0.052 \\
MTX & 0.03 & TNFi & 0.27 & $10.8(1.5-80.1)$ & 0.019 \\
MTX & 0.03 & ETA & 0.11 & $4.4(0.5-35.8)$ & 0.165 \\
MTX & 0.03 & TNF-antibodies & 0.75 & $42.9(5.8-317)$ & 0.0002 \\
ETA & 0.11 & TNF-antibodies & 0.75 & $6.8(2.8-16.5)$ & 0.00002 \\
MTX & 0.03 & ADA & 0.69 & $27.4(3.6-2209)$ & 0.001 \\
Non-TNFi & 0.63 & ADA & 0.69 & $1.1(0.4-2.7)$ & 0.847 \\
Non-TNFi & 0.63 & ETA & 0.11 & $0.2(0.1-0.5)$ & 0.0012 \\
ETA & 0.11 & ADA & 0.69 & $6.2(0.5-15.6)$ & 0.0001 \\
\hline
\end{tabular}

Conclusion: Our findings demonstrate a higher incidence of psoriasis in monoclonal-antibody-TNFi-treated JIA-patients, whereas in Etanercept-treated JIA patients no significant increase was detected. On average, psoriasis-manifestation occurred more than two years after treatment-initiation. Teenage females with ANA-positivity were most often affected.

Disclosure of Interests: Angela Zimmer: None declared, Ariane Klein Consultant of: Celgene, Gerd Horneff Grant/research support from: AbbVie, Chugai, Merck Sharp \& Dohme, Novartis, Pfizer, Roche, Speakers bureau: AbbVie, Bayer, Chugai, Merck Sharp \& Dohme, Novartis, Pfizer, Roche DOI: 10.1136/annrheumdis-2020-eular.4883

\section{\begin{tabular}{|l|l}
\hline SAT0492 RAPID IMPROVEMENT AND ACHIEVEMENT OF \\
\hline
\end{tabular} INACTIVE DISEASE IN CHILDREN WITH SYSTEMIC JUVENILE IDIOPATHIC ARTHRITIS UNDER 4 YEARS OF AGE DEPENDING ON DISEASE DURATION ON TOCILIZUMAB TREATMENT}

E. Krekhova ${ }^{1}$, E. Alexeeva ${ }^{1,2}$, T. Dvoryakovskaya ${ }^{1,2}, \mathrm{~K}$ Isaeva $^{2}$,

A. Chomakhidze ${ }^{2}$, E. Chistyakova $^{1,2}$, O. Lomakina ${ }^{2}$, R. Denisova ${ }^{2}$,

A. Mamutova ${ }^{2}$, A. Fetisova ${ }^{2}$, M. Gautier ${ }^{2}$, D. Vankova ${ }^{2}$, M. Shingarova ${ }^{1}$,

Table 1

\begin{tabular}{|c|c|c|c|c|c|c|c|c|}
\hline N/PY & $\begin{array}{l}\text { TotalCohort }^{\star} \\
4792 / 13519\end{array}$ & $\begin{array}{c}\text { All TNFi } \\
4149 / 8437\end{array}$ & $\begin{array}{c}\text { ADA } \\
1105 / 1859\end{array}$ & $\begin{array}{c}\text { ETA } \\
2824 / 6307\end{array}$ & $\begin{array}{c}\text { GOL } \\
144 / 145\end{array}$ & $\begin{array}{c}\text { INF } \\
76 / 126\end{array}$ & $\begin{array}{c}\text { Non-TNFi } \\
676 / 1112\end{array}$ & $\begin{array}{l}\text { MTX only } \\
1692 / 3971\end{array}$ \\
\hline Psoriasis events/rate(\%) & $31 / 0.6$ & $23 / 0.6$ & $13 / 1.2$ & $7 / 0.2$ & $2 / 1.4$ & $1 / 1.3$ & $7 / 0.6$ & $1 / 0.1$ \\
\hline $\mathrm{Pso}^{\star \star} / 100 \mathrm{PY}(95 \% \mathrm{Cl})$ & $\begin{array}{c}0.2 \\
(0.2-0.3)\end{array}$ & $\begin{array}{c}0.3 \\
(0.2-0.4)\end{array}$ & $\begin{array}{c}0.7 \\
(0.4-1.2)\end{array}$ & $\begin{array}{c}0.1 \\
(0.1-0.2)\end{array}$ & $\begin{array}{c}1.4 \\
(0.3-5.5)\end{array}$ & $\begin{array}{c}0.8 \\
(0.1-5.6)\end{array}$ & $\begin{array}{c}1.0 \\
(0.5-2.1)\end{array}$ & $\begin{array}{c}0.03 \\
(0.004-0.2)\end{array}$ \\
\hline $\begin{array}{l}\text { Age at event } \\
\text { Mean } \pm S D\end{array}$ & $\begin{array}{c}13.9 \\
( \pm 3.1)\end{array}$ & $\begin{array}{c}13.9 \\
( \pm 3.2)\end{array}$ & $\begin{array}{c}14.0 \\
( \pm 3.0)\end{array}$ & $\begin{array}{c}13.0 \\
( \pm 4.0)\end{array}$ & $\begin{array}{c}15.1 \\
( \pm 3.4)\end{array}$ & 16.4 & $\begin{array}{c}13.4 \\
( \pm 3.1)\end{array}$ & 17.3 \\
\hline Female & $\begin{array}{c}24 \\
(77 \%)\end{array}$ & $\begin{array}{c}18 \\
(78 \%)\end{array}$ & $\begin{array}{c}10 \\
(77 \%)\end{array}$ & $\begin{array}{c}5 \\
(71 \%)\end{array}$ & $\begin{array}{c}2 \\
(100 \%)\end{array}$ & $\begin{array}{c}1 \\
(100 \%)\end{array}$ & $\begin{array}{c}5 \\
(71 \%)\end{array}$ & $\begin{array}{c}1 \\
(100 \%)\end{array}$ \\
\hline ANA positive & $\begin{array}{c}22 \\
(71 \%)\end{array}$ & $\begin{array}{c}16 \\
(70 \%)\end{array}$ & $\begin{array}{c}10 \\
(77 \%)\end{array}$ & $\begin{array}{c}4 \\
(57 \%)\end{array}$ & $\begin{array}{c}1 \\
(50 \%)\end{array}$ & $\begin{array}{c}1 \\
(100 \%)\end{array}$ & $\begin{array}{c}5 \\
(71)\end{array}$ & $\begin{array}{c}1 \\
(100 \%)\end{array}$ \\
\hline $\begin{array}{l}\text { Treatment duration until } \\
\text { event (years) Mean } \pm S D\end{array}$ & $\begin{array}{l}2.2 \\
\pm 1.8\end{array}$ & $\begin{array}{c}2.4 \\
\pm 2.3\end{array}$ & $\begin{array}{l}2.2 \\
\pm 1.4\end{array}$ & $\begin{array}{l}2.8 \\
\pm 2.2\end{array}$ & $\begin{array}{l}0.6 \\
\pm 0.5\end{array}$ & 3.8 & $\begin{array}{c}1.4 \\
\pm 2.0\end{array}$ & 5.6 \\
\hline $\begin{array}{l}\text { Concomitant } \\
\text { MTX }\end{array}$ & $\begin{array}{c}11 \\
(36 \%)\end{array}$ & $\begin{array}{c}6 \\
(26 \%)\end{array}$ & $\begin{array}{c}3 \\
(23 \%)\end{array}$ & $\begin{array}{c}2 \\
(29 \%)\end{array}$ & $\begin{array}{c}1 \\
(50 \%)\end{array}$ & 0 & $\begin{array}{c}4 \\
(57 \%)\end{array}$ & $\begin{array}{c}1 \\
(100 \%)\end{array}$ \\
\hline $\begin{array}{l}\text { Concomitant } \\
\text { Steroids }\end{array}$ & $\begin{array}{c}4 \\
(13 \%)\end{array}$ & $\begin{array}{c}2 \\
(9 \%)\end{array}$ & 0 & $\begin{array}{c}1 \\
(14 \%)\end{array}$ & 0 & $\begin{array}{c}1 \\
(100 \%)\end{array}$ & $\begin{array}{c}2 \\
(29 \%)\end{array}$ & 0 \\
\hline JADAS10 Median [IQR] $]^{\star \star *}$ & $\begin{array}{c}2.5 \\
(0.6-8.8)\end{array}$ & $\begin{array}{c}4.2 \\
(1.0-11.0)\end{array}$ & $\begin{array}{c}2.0 \\
(0.6-10.1)\end{array}$ & $\begin{array}{c}5.8 \\
(1.5-11.4)\end{array}$ & 18.0 & 6.6 & $\begin{array}{c}1.3 \\
(0.8-3.4)\end{array}$ & 1.7 \\
\hline
\end{tabular}

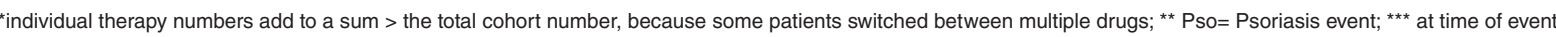

